\title{
Capsaicin prevents degeneration of dopamine neurons by inhibiting glial activation and oxidative stress in the MPTP model of Parkinson's disease
}

\author{
Young C Chung ${ }^{1,8}$, Jeong Y Baek ${ }^{2,8}$, Sang R Kim ${ }^{3,4}$, Hyuk W Ko${ }^{1}$, Eugene Bok ${ }^{4}$, Won-Ho Shin ${ }^{5}$, \\ So-Yoon Won ${ }^{6}$ and Byung K Jin ${ }^{2,7}$
}

The effects of capsaicin (CAP), a transient receptor potential vanilloid subtype 1 (TRPV1) agonist, were determined on nigrostriatal dopamine (DA) neurons in the 1-methyl-4-phenyl-1,2,3,6-tetrahydropyridine (MPTP) mouse model of Parkinson's disease (PD). The results showed that TRPV1 activation by CAP rescued nigrostriatal DA neurons, enhanced striatal DA functions and improved behavioral recovery in MPTP-treated mice. CAP neuroprotection was associated with reduced expression of proinflammatory cytokines (tumor necrosis factor- $\alpha$ and interleukin-1 $\beta$ ) and reactive oxygen species/reactive nitrogen species from activated microglia-derived NADPH oxidase, inducible nitric oxide synthase or reactive astrocyte-derived myeloidperoxidase. These beneficial effects of CAP were reversed by treatment with the TRPV1 antagonists capsazepine and iodo-resiniferatoxin, indicating TRPV1 involvement. This study demonstrates that TRPV1 activation by CAP protects nigrostriatal DA neurons via inhibition of glial activation-mediated oxidative stress and neuroinflammation in the MPTP mouse model of PD. These results suggest that CAP and its analogs may be beneficial therapeutic agents for the treatment of PD and other neurodegenerative disorders that are associated with neuroinflammation and glial activation-derived oxidative damage.

Experimental \& Molecular Medicine (2017) 49, e298; doi:10.1038/emm.2016.159; published online 3 March 2017

\section{INTRODUCTION}

Parkinson's disease (PD) is characterized by the progressive degeneration of nigrostriatal dopamine (DA) neurons, ${ }^{1}$ which is associated with motor dysfunctions such as slowness of movement, resting tremor and rigidity. ${ }^{1-3}$ Although the exact cause of PD remains unclear, both clinical and experimental data demonstrate that reactive glia can produce $\mathrm{NADPH}$ oxidase-derived reactive oxygen species (ROS) and/or myeloperoxidase (MPO)-derived reactive nitrogen $s$ pecies (RNS), which result in oxidative damage to DA neurons. ${ }^{4-6}$ Moreover, proinflammatory neurotoxic mediators, such as tumor necrosis factor (TNF)- $\alpha$, interleukin (IL)-1 $\beta$ and inducible nitric oxide synthase (iNOS), are increased in the substantia nigra (SN) of PD patients and in the 1-methyl-4-phenyl-1,2,3,6-tetrahydropyridine (MPTP) mouse model..$^{-9}$

Transient receptor potential receptor vanilloid type 1 (TRPV1), the capsaicin (CAP) receptor, is a nonselective cation channel that causes pain and is highly expressed in sensory neurons. ${ }^{10}$ TRPV1 is also present in the brain, suggesting that this receptor has an important role in the central nervous system. The activation of TRPV1 was found to modulate neuronal function, ${ }^{11,12}$ control motor behavior ${ }^{13,14}$ and regulate neuroinflammation. ${ }^{15}$

${ }^{1}$ College of Pharmacy, Dongguk University, Goyang, Republic of Korea; ${ }^{2}$ Department of Neuroscience, Graduate School, Kyung Hee University, Seoul, Republic of Korea; ${ }^{3}$ School of Life Sciences, BK21 plus KNU Creative BioResearch Group, Kyungpook National University, Daegu, Republic of Korea; ${ }^{4}$ Brain Science and Engineering Institute, Kyungpook National University, Daegu, Republic of Korea; ${ }^{5}$ Predictive model Research Center, Korea Institute of Toxicology, Korea Research Institute of Chemical Technology, Daejeon, Republic of Korea; ${ }^{6}$ Department of Biochemistry and Signaling Disorder Research Center, College of Medicine, Chungbuk National University, Cheongju, Republic of Korea and ${ }^{7}$ Department of Biochemistry and Molecular Biology, Neurodegeneration Control Research Center, School of Medicine Kyung Hee University, Seoul, Republic of Korea

8These authors contributed equally to this work.

Correspondence: Dr W-H Shin, Predictive Model Research Center, Korea Institute of Toxicology, Korea Research Institute of Chemical Technology, Daejeon 34114, Republic of Korea.

E-mail: whshin@kitox.re.kr

or Dr S-Y Won, Department of Biochemistry and Signaling Disorder Research Center, College of Medicine, Chungbuk National University, Cheongju 361763, Republic of Korea.

Email: sywon@chungbuk.ac.kr

or Professor BK Jin, Department of Neuroscience, Graduate School, Kyung Hee University, Seoul 130-701, Republic of Korea.

E-mail: bkjin@khu.ac.kr

Received 1 March 2016; revised 6 July 2016; accepted 24 August 2016 
Moreover, CAP regulates neurotransmitter release from nigral slices, ${ }^{16}$ produces hypokinesia in parallel with a decrease in the activity of nigrostriatal DA neurons, ${ }^{17,18}$ and modulates the effects of high levels of L-DOPA on motor activity in reserpine-treated Parkinsonian rats, ${ }^{19}$ suggesting that TRPV1 has a functional role in nigrostriatal DA neurons. The current study sought to determine whether TRPV1 activation by CAP could rescue nigrostriatal DA neurons by inhibiting glial activation and reactive glia-derived oxidative stress in the MPTP mouse model of PD.

\section{MATERIALS AND METHODS}

\section{Chemicals}

The following chemicals were purchased: capsazepine (CZP; Tocris Cookson, Ellisville, MO, USA), iodo-resiniferatoxin (I-RTX; Tocris Cookson, Ellisville, MO, USA), CAP (Sigma, St Louis, MO, USA) and MPTP (Sigma). CAP, CZP and I-RTX were dissolved in ethanol and Tween-80, and then diluted with phosphate-buffered saline (PBS; 1:1:8, ethanol:Tween-80:PBS)

\section{Animals and drug treatments}

The animal protocols and guidelines were established and approved by Kyung Hee University. All experiments were conducted according to the relevant national guidelines. Eight- to ten-week-old male C57BL/6 mice (23-25 g, Charles River Breeding Laboratory, Yokohama, Japan) were used for MPTP intoxication. MPTP $\left(20 \mathrm{mg} \mathrm{kg}^{-1}\right.$, free base; Sigma) was dissolved in PBS and then intraperitoneally injected into mice for four times at 2-h intervals. Various doses of the CAP $\left(0.01,0.1,0.5,1\right.$ and $\left.2.5 \mathrm{mg} \mathrm{kg}^{-1}\right)$ were intraperitoneally administered for 1 day starting $30 \mathrm{~min}$ before MPTP injection. The TRPV1 antagonists CZP and I-RTX $\left(1 \mathrm{mg} \mathrm{kg}^{-1}\right)$ were injected into the peritoneum for $30 \mathrm{~min}$ before the TRPV1 agonist injections. Control mice were injected with the vehicle (Supplementary Figure 1).

\section{Immunohistochemistry}

For immunohistochemistry, mouse brain tissues were prepared as previously described. ${ }^{20,21}$ Sections were washed with PBS, then permeabilized with $0.2 \%$ Triton X-100 dissolved in $0.1 \mathrm{M}$ PBS containing $1 \%$ bovine serum albumin at room temperature for $30 \mathrm{~min}$. Tissue sections were labeled with rabbit anti-tyrosine hydroxylase (TH; 1:2000; Pel-Freez, Brown Deer, WI, USA), rat antimacrophage Ag complex-1 (MAC-1; 1:200; Serotec, Oxford, UK), rat anit-CD68 (ED-1; 1:1000; Serotec), mouse anti-glial fibrillary acidic protein (GFAP; 1:5000; Neuromics, Edina, MN, USA), rabbit antiMPO (1:500; Thermo Scientific, Fremont, CA, USA) and mouse anti8-hydroxy-29-deoxyguanosine (8-OHdG; 1:200; JaICA, Shizuoka, Japan). After overnight incubation, sections were washed and incubated with proper biotinylated secondary antibodies for $1 \mathrm{~h}$. After washing, the sections were stained with an $\mathrm{ABC}$ kit according to the manufacturer's instructions. Stained samples were mounted on gelatin-coated slides and analyzed under a bright-field microscope (Nikon, Tokyo, Japan). To determine the immunoreactivity of the 8-OHdG-labeled area, three or four images of the SN region were obtained, thresholded using Image J (NIH, Bethesda, MD, USA), quantified and normalized by the value of the control group.

\section{Stereological cell counts}

As previously described, ${ }^{20,21}$ the total number of TH-positive neurons or MPO-ip cells was counted in the various animal groups based on the optical fractionator method using a bright-field microscope (Olympus Optical, BX51, Tokyo, Japan) and Stereo Investigator software (MBF Bioscience, Williston, VT, USA). The actual counting of TH-labeled cells was performed within the boundaries of the optical fractionator. The estimated total number of cells was calculated according to the optical fractionator software. More than total 300 points overall of the sections of each specimen were analyzed.

\section{Densitometric analysis}

As previously described, ${ }^{20-22}$ the TH-labeled sections were imaged using a light microscope (Zeiss Axioskop, Oberkochen, Germany) coupled to a video camera (MegaPlus model 1.4i; Kodak, New York, NY, USA) and analyzed using an Image-Pro Plus system (Ver 4.0; Media Cybermetrics, Silvers Spring, MD, USA). To normalize the images for differences in background illumination, the average of the background density readings from the corpus callosum was subtracted from the average of the density for each section analyzed. Then, the average of all of the sections of each animal was calculated separately before the data were statistically processed.

\section{Rotarod test}

To measure forelimb and hindlimb motor dysfunction, mice were placed on a Rotarod system (five-lane accelerating rotarod; Ugo Basile, Comerio, Italy) as previously described. ${ }^{20-22}$ For the training session, each mouse was placed onto the rotating rod (10 r.p.m.) for $20 \mathrm{~min}$ for 7 consecutive days before the MPTP treatment. Seven days from the last MPTP treatment, the mice were placed in a separate compartment on the rotating rod and tested at 20 r.p.m. for $20 \mathrm{~min}$. The latency to fall from the rotating rod within this time period was automatically recorded by magnetic trip plates.

\section{Measurement of DA levels (high-performance liquid chromatography analyses)}

As described, ${ }^{20}$ the levels of DA in the striatum (STR) were measured using high-performance liquid chromatography consisting of an autosampler (Waters 717 Plus Autosampler, Waters Division, Milford, MA, USA) and an ESA CoulochemII electrochemical detector equipped with an mBondapak C18 column $(3.9 \times 300 \mathrm{~mm} \times 10 \mu \mathrm{m}$; ESA, Chelmsford, MA, USA). Dissected striatal tissues were homogenized with $0.1 \mathrm{M}$ perchloric acid and $0.1 \mathrm{~mm}$ EDTA, and centrifuged at 900 r.p.m. for $20 \mathrm{~min}$. All supernatants were collected and eluted with a mobile phase for catecholamine analysis (Chromosystems, Munich, Germany), and the column temperature was maintained at $4{ }^{\circ} \mathrm{C}$. The contents of DA in the STR were analyzed using a commercially available program (Breeze; Waters Corp., Tokyo, Japan).

\section{Measurement of MPTP and 1-methyl-4-phenyl-pyridinium levels in the STR}

For sample preparation, dissected striatal tissues were collected, sonicated and immediately centrifuged at 9000 r.p.m. for $20 \mathrm{~min}$, and the supernatant was saved. As described previously, ${ }^{21}$ MPTP and 1-methyl-4-phenyl-pyridinium $\left(\mathrm{MPP}^{+}\right)$levels were analyzing with a liquid chromatography electrospray ionization mass spectrometry system composed of three Agilent model G1311A HPLC quaternary pumps (Palo Alto, CA, USA), a G1313A standard autosampler and a G1316A thermostated column compartment. The samples were eluted through a $150 \mathrm{~mm} \times 1.5 \mathrm{~mm}$ inner diameter, $4 \mu \mathrm{m}$ Zorbax Eclipse XDB-C18 (Palo Alto, CA, USA). All samples were normalized for protein content, which was determined spectrophotometrically using a Bio-Rad protein assay kit (Bio-Rad, Hercules, CA, USA). 
In situ detection of $\mathrm{O}_{2}$ - and $\mathrm{O}_{2}$-derived oxidants

As previously described, ${ }^{21,22}$ production of $\mathrm{O}_{2^{-}}$and $\mathrm{O}_{2}$-derived oxidants was assessed by detecting ethidine labeled by hydroethidine histochemistry. ${ }^{21,22}$ Hydroethidine was administered intraperitoneally at 3 days after the last MPTP treatment. Brain samples were collected $15 \mathrm{~min}$ after hydroethidine injection and then fixed with $4 \%$ paraformaldehyde in $0.1 \mathrm{M}$ phosphate buffer. After fixation, the brains were sectioned, mounted on gelatin-coated slides and counterstained with 4,6-diamidino-2-phenylindole to detect cellular nuclei. All images
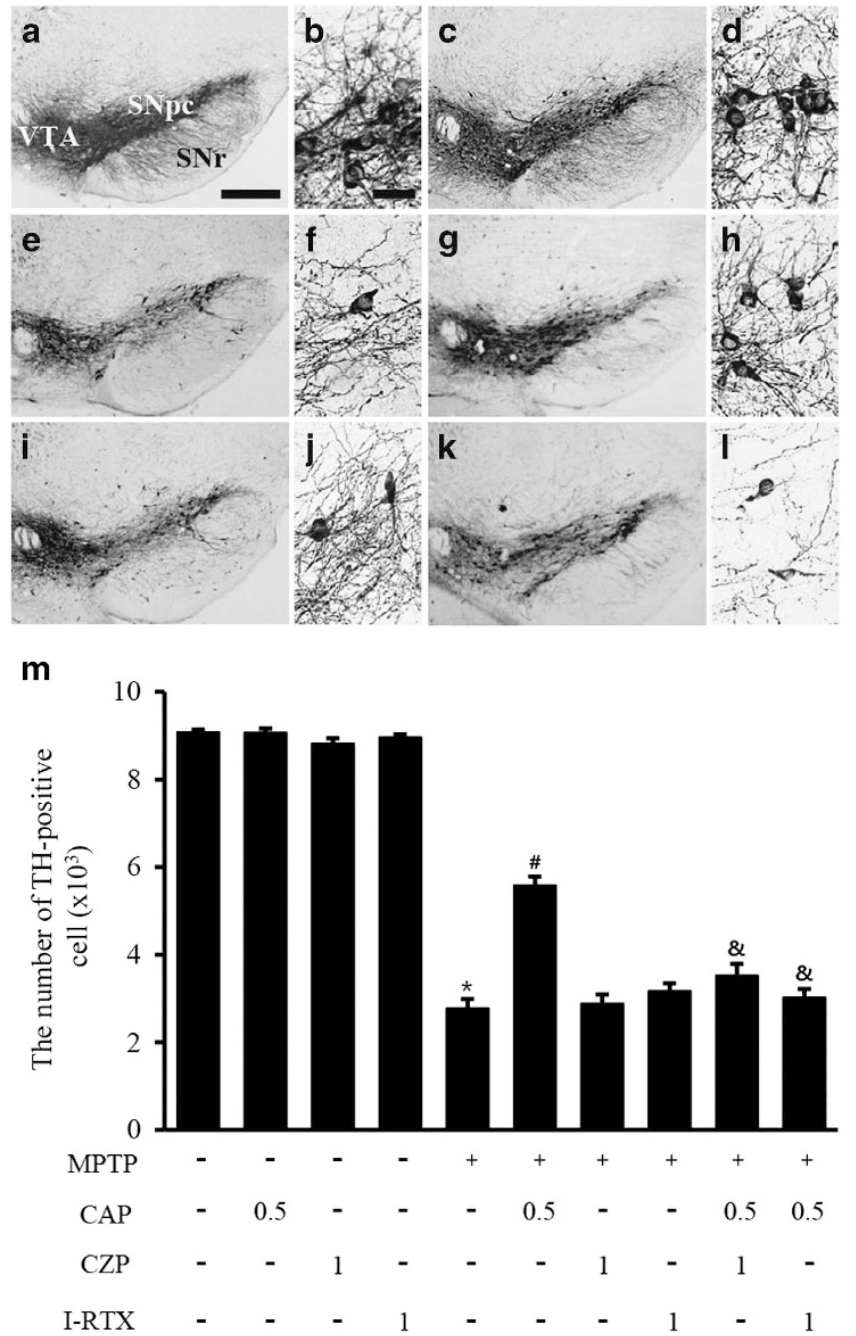

Figure 1 Capsaicin (CAP) attenuates 1-methyl-4-phenyl-1,2,3,6tetrahydropyridine (MPTP) neurotoxicity in the substantia nigra (SN) in vivo. Seven days after the last MPTP injection, animals were killed, and their brain tissues were processed for immunostaining with a tyrosine hydroxylase (TH) antibody for dopamine (DA) neurons: phosphate-buffered saline (PBS) (a, b), CAP $\left(0.5 \mathrm{mg} \mathrm{kg}^{-1}\right)$ alone (c, d), MPTP (e, f), MPTP+CAP (g, h), MPTP + CAP+capsazepine (CZP) (i, j) and MPTP+CAP+iodo-resiniferatoxin $(\mathrm{I}-\mathrm{RTX})(\mathbf{k}, \mathbf{I})$. (m) The numbers of TH-positive cells in the SNpc. Bars represent the means \pm s.e.m. of five to six animals per group. ${ }^{*} P<0.001$ significantly different from control, ${ }^{\#} P<0.001$, significantly different from MPTP. \& $P<0.001$ significantly different from MPTP and CAP. SNpc, substantia nigra pars compacta; SNr, substantia nigra reticulata; VTA, ventral tegmental area. Scale bars, $300 \mu \mathrm{m}(\mathbf{a}, \mathbf{c}, \mathbf{e}, \mathbf{g}, \mathbf{i}, \mathbf{k}) ; 50 \mu \mathrm{m}(\mathbf{b}, \mathbf{d}, \mathbf{f}, \mathbf{h}, \mathbf{j}, \mathbf{k})$. were acquired using a confocal microscope (Carl Zeiss, Oberkochen, Germany) in the frame scan mode with the same exposure time.

\section{Immunoprecipitation and western blotting}

As previously described, ${ }^{22} \mathrm{SN}$ samples were homogenized and collected, and the supernatants were centrifuged for $20 \mathrm{~min}$ at $13000 \mathrm{~g}$ at $4{ }^{\circ} \mathrm{C}$. The total protein $(100 \mu \mathrm{g})$ was immunoprecipitated with anti-p47phox antibody (Ab; $1.0 \mu \mathrm{g}$; Santa Cruz Biotechnology, Dallas, TX, USA). The immunoprecipitated proteins were separated on an SDS-polyacrylamide gel electrophoresis gel and were then electro-transferred onto polyvinylidene difluoride membranes and blocked for $30 \mathrm{~min}$ at room temperature with tris-buffered saline. The immunodetection of target proteins was performed using specific monoclonal antibodies as follows: anti-phosphoserine Ab (1:5000; Invitrogen Life Technologies, Rockville, MD, USA), anti-p47phox Ab (1:1000; Santa Cruz Biotechnology) and anti-gp91phox Ab (1:4000; BD Biosciences, CA, USA). For semiquantitative analyses, Ab immunoreactivity on the membrane was analyzed using a Computer Imaging Device (Seoul, South Korea) and the accompanying software (Fujifilm, Seoul, South Korea).

\section{Reverse transcription PCR}

After injection of the TRPV1 agonist and/or antagonists, the mice were killed at $24 \mathrm{~h}$ after injection of MPTP, and the SN tissues were rapidly removed. The total RNA was extracted using RNAzol B (Tel-Test, Friendwood, TX, USA) and converted to complementary DNA using Superscript II reverse transcriptase (Life Technologies, Rockville, MD, USA) according to the manufacturer's instructions. PCR was performed with the following primer sequences: 5'-CTGCTGGTGG TGACAAGCACATTT-3' (forward) and 5'-ATGTCATGAGCAAAGG CGCAGAAC-3' (reverse) for iNOS; 5'-GCGACGTGGAACTGGCAGA AGAG-3' (forward) and $5^{\prime}$-TGAGAGGGAGGCCATTTGGGAAC-3' (reverse) for TNF- $\alpha$; $5^{\prime}$-GCAACTGTTCCTGAACTCAACT-3' (forward) and $5^{\prime}$-ATCTTTTGGGGTCCGTCAACT-3' (reverse) for IL-1 $\beta$; and $5^{\prime}$-GCAATTATTCCCCATGAACG-3' (forward) and $5^{\prime}$-GGCCTCACTAAACCATCCAA-3' (reverse) for $18 \mathrm{~s}$ ribosomal RNA. Annealing was performed at $56^{\circ} \mathrm{C}$ for $30 \mathrm{~s}$ (for iNOS, TNF- $\alpha$ and IL-1 $\beta$ ), and PCR reaction was ended with a final extension at $72{ }^{\circ} \mathrm{C}$ for $90 \mathrm{~s}$. The PCR products were separated by electrophoresis on $1.5 \%$ agarose gels, visualized with ethidium bromide and photographed. For semiquantitative analyses, the photographs were scanned using a Computer Imaging Device and the accompanying software (Fujifilm).

\section{Statistical analysis}

All data are represented as the means \pm s.e.m. Statistical significance $(P<0.05$ for all analyses) was assessed using analysis of variance (Instat 3.05, GraphPad, San Diego, CA, USA) followed by StudentNewman-Keuls analyses.

\section{RESULTS}

CAP rescues nigrostriatal DA neurons in vivo in MPTP-

lesioned mice

Similar to our recent reports, ${ }^{21,22}$ the TH immunohistochemical analysis revealed that MPTP exerted significant neurotoxicity on TH-positive cell bodies in the SN (Figure 1e and $\mathrm{f}$ ) and its fibers in the STR (Figure 2c) at 7 days compared with control PBS (intact)-treated mice (Figures $1 \mathrm{a}, \mathrm{b}$ and $2 \mathrm{a}$ ). The results of the stereological counts and densitometric 


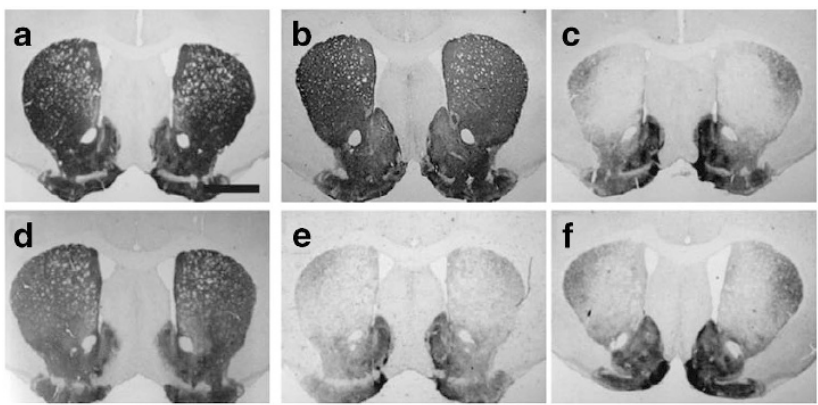

g
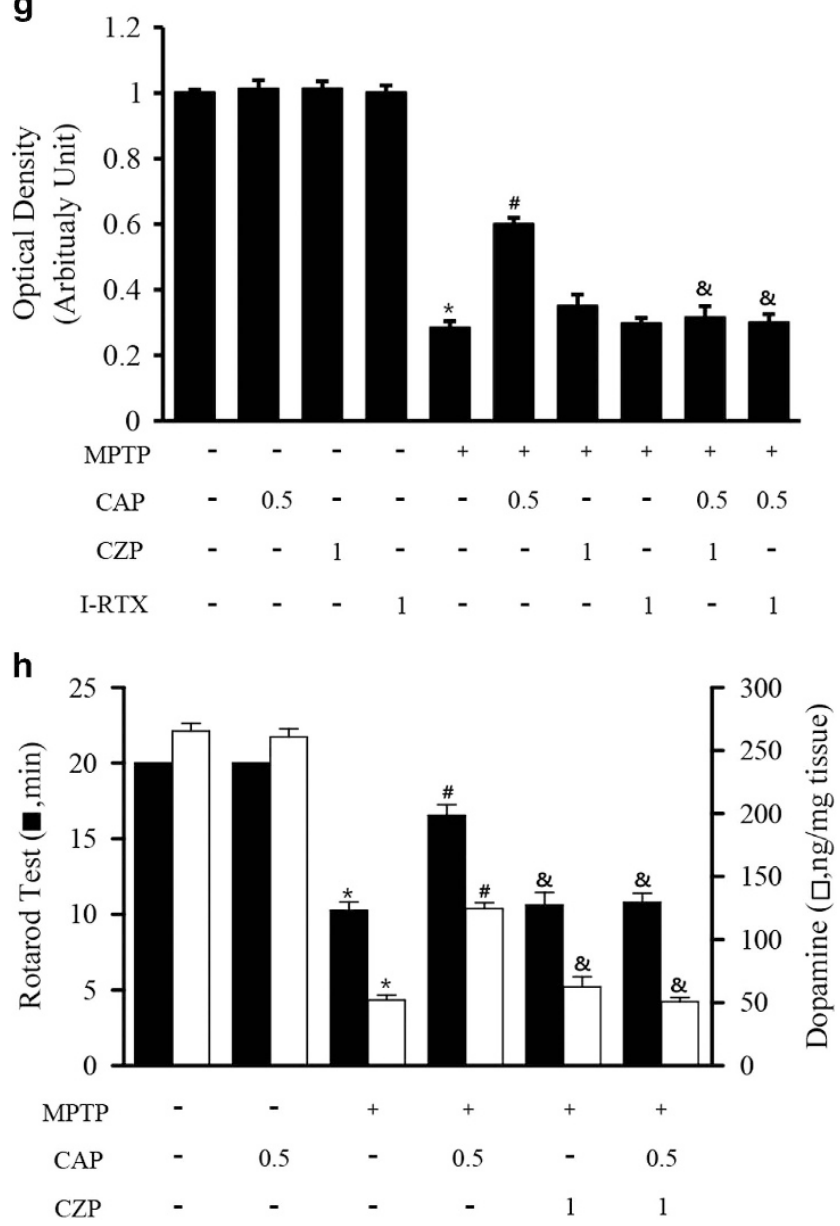

Figure 2 Capsaicin (CAP) prevents 1-methyl-4-phenyl-1,2,3,6tetrahydropyridine (MPTP) neurotoxicity in the striatum (STR) in vivo. The STR tissues obtained from the same animals as used in Figure 1 were immunostained with a $\mathrm{TH}$ antibody for dopamine (DA) fibers: phosphate-buffered saline (PBS) (a), CAP alone (b), MPTP (c), MPTP+CAP (d), MPTP+CAP+CZP (e) or MPTP+CAP + I-RTX (f). (g) The optical density of the TH-positive fibers in the STR. ${ }^{*} P<0.001$ significantly different from control, ${ }^{\#} P<0.001$, significantly different from MPTP. \& $P<0.001$ significantly different from MPTP and CAP. Scale bars, $500 \mu \mathrm{m}$. (h) After the rotarod test, striatal tissues were dissected from the animals to determine the dopamine level in the striatum. Bars represent the means \pm s.e.m. of five to six animals per group. ${ }^{*} P<0.001$, significantly different from control, ${ }^{\#} P<0.001$, significantly different from MPTP. \& $P<0.001$, significantly different from MPTP and CAP (analysis of variance and Student-Newman-Keuls analysis). analyses showed a significant reduction in the number of $\mathrm{TH}$-positive cells by $72 \%$ in the SN (Figure $1 \mathrm{~m}$ ) and the density of TH-positive fibers by $77 \%$ in the STR (Figure $2 \mathrm{~g}$ ) in the MPTP-treated mice compared with PBS-treated control mice.

To explore the effects of CAP on the MPTP-induced neurotoxicity of nigrostriatal DA neurons, various doses of CAP $\left(0.1-2.5 \mathrm{mg} \mathrm{kg}^{-1}\right.$, intraperitoneal) were administered 30 min before the first MPTP injection. TH immunohistochemistry revealed dose-dependent CAP neuroprotection on nigrostriatal DA neurons at 7 days post MPTP treatment (Supplementary Table 1). Based on this result, a $0.5 \mathrm{mg} \mathrm{kg}^{-1}$ dose of CAP was chosen for further studies and also effectively increased the number of TH-positive cells by $38 \%$ (Figure 1g,h and $\mathrm{m} ; P<0.01)$ and density of TH-positive fibers by $36 \%$ (Figure $2 \mathrm{~d}$ and g; $P<0.001$ ). Neuroprotection afforded by CAP was almost completely reversed by pretreatment with either of the TRPV1 antagonists, CZP (Figures 1i,j,m and 2e,g) or I-RTX (Figures $1 \mathrm{k}-\mathrm{m}, 2 \mathrm{f}$ and $\mathrm{g}$ ), indicating TRPV1 involvement. CAP alone did not affect the TH immunoreactivity in the SN (Figure $1 \mathrm{c}$ and d) and STR (Figure 2b).

Next, we hypothesized that CAP neuroprotection may result from the altered metabolism of MPTP to $\mathrm{MPP}^{+}$in vivo. Because striatal levels of $\mathrm{MPP}^{+}$, an active toxic metabolite of MPTP, represent MPTP neurotoxicity, ${ }^{23,24}$ we measured the difference in striatal MPTP and MPP ${ }^{+}$levels $2 \mathrm{~h}$ after the last injection of MPTP in the presence or absence of CAP (Table 1). The results showed no substantial difference in the striatal levels of MPTP and $\mathrm{MPP}^{+}$, ruling out the possibility that CAP affects MPTP conversion to $\mathrm{MPP}^{+}$in vivo.

\section{CAP improves motor behavior by restoring striatal DA depletion in MPTP mice}

To determine CAP effects on behavioral recovery in MPTPlesioned mice, the latency to falling on the rotarod apparatus was analyzed at 7 days after the last MPTP injection in the absence or presence of CAP. ${ }^{21,22}$ In MPTP-lesioned mice, robust motor deficits were evident, as determined by decreased sustained rotarod time to $9.62 \pm 0.53 \mathrm{~min}$, a $53 \%$ decrease compared with the PBS-treated control $(P<0.001$; Figure $2 \mathrm{~h})$. In the CAP-treated MPTP-lesioned mice, the latency to fall was significantly increased up to $16.27 \pm 0.45 \mathrm{~min}$ $(P<0.001$; Figure $2 \mathrm{~h})$, which is indicative of behavioral rescue. This behavioral improvement was almost completely reversed by pretreatment with CZP $(10.12 \pm 0.42 \mathrm{~min}$, $P<0.001$; Figure $2 \mathrm{~h}$ ).

In parallel with motor recovery, CAP produced an increase in striatal DA function. The high-performance liquid chromatography analysis revealed an MPTP-induced decrease in striatal DA levels by $72 \%$ compared with the PBS controls (Figure 2h). Compared with MPTP only, CAP resulted in a $42 \%$ increase in striatal DA levels $(P<0.001$; Figure $2 \mathrm{~h})$. The increase in STR DA levels was almost completely reversed by CZP pretreatment $(P<0.001$; Figure $2 \mathrm{~h})$. CAP or CZP alone did not affect the latency to fall or STR DA levels. 
CAP inhibits microglial activation and the expression of proinflammatory mediators in the $\mathrm{SN}$ in vivo Because activated microglia can cause degeneration of DA neurons in the MPTP model, ${ }^{21,22,25}$ we wondered whether CAP could exert neuroprotection via inhibition of microglial

Table 1 The effects of TRPV1 agonist on MPTP and MPP+ levels ( $\mu \mathrm{g} \mathrm{mg}^{-1}$ protein) in the striata of $\mathrm{C} 57 \mathrm{BL} 6$ mice

\begin{tabular}{cccc}
\hline & Con & MPTP & MPTP+capsaicin \\
\hline MPTP & 0 & $2.5 \pm 0.9$ & $2.7 \pm 0.5$ \\
MPP $^{+}$ & 0 & $3.4 \pm 0.2$ & $3.4 \pm 1.2$
\end{tabular}

Abbreviation: MPTP, 1-methyl-4-phenyl-1,2,3,6-tetrahydropyridine.

For capsaicin treatment, mice received a single injection/days of capsaicin (1 $\mathrm{mg} \mathrm{kg}^{-1}$ body weight) into the peritoneum, beginning at $30 \mathrm{~min}$ before first MPTP injection. After pretreatment, mice received four intraperitoneal injections of MPTP ( $20 \mathrm{mg} \mathrm{kg}^{-1}$ body weight) at $2 \mathrm{~h}$ intervals. Striatal tissues were removed at $2 \mathrm{~h}$ after the last MPTP injection, and MPTP and MPP+ levels were measured by liquid chromatography electrospray ionization mass spectrometry (LC/ESI-

MASS). Note that there are no changes of striatal MPP ${ }^{+}$levels in the absence and presence of TRPV1 agonists.
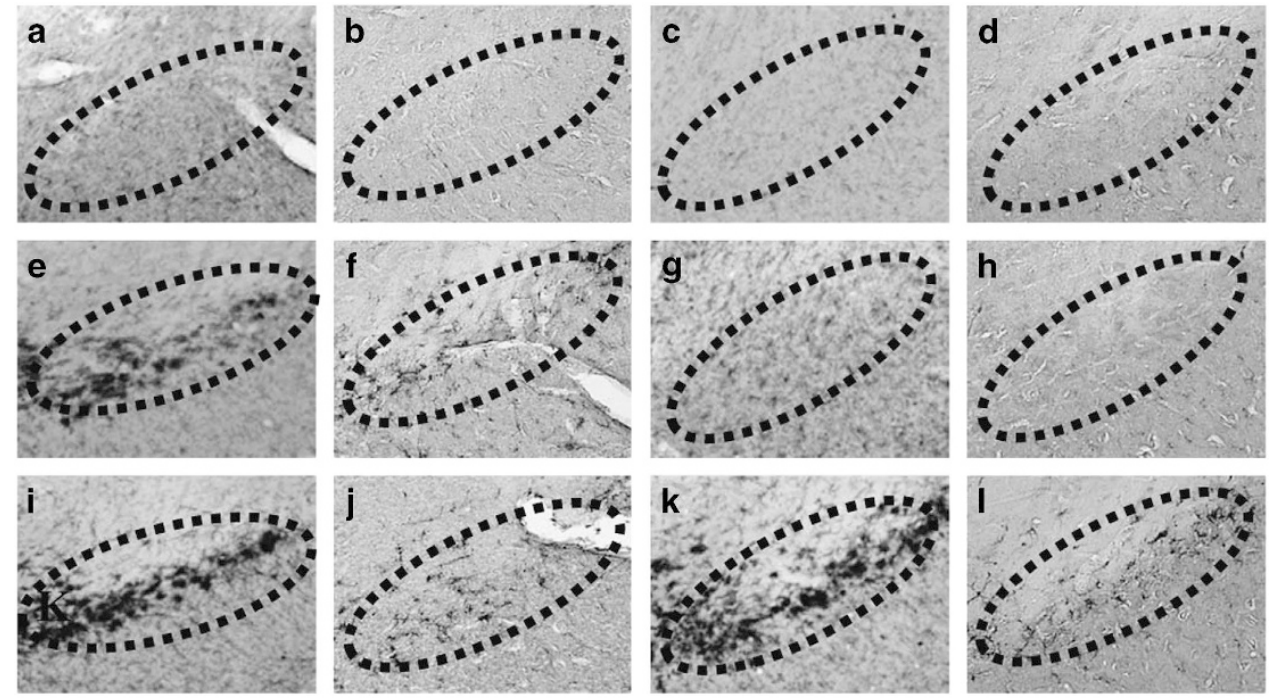

m
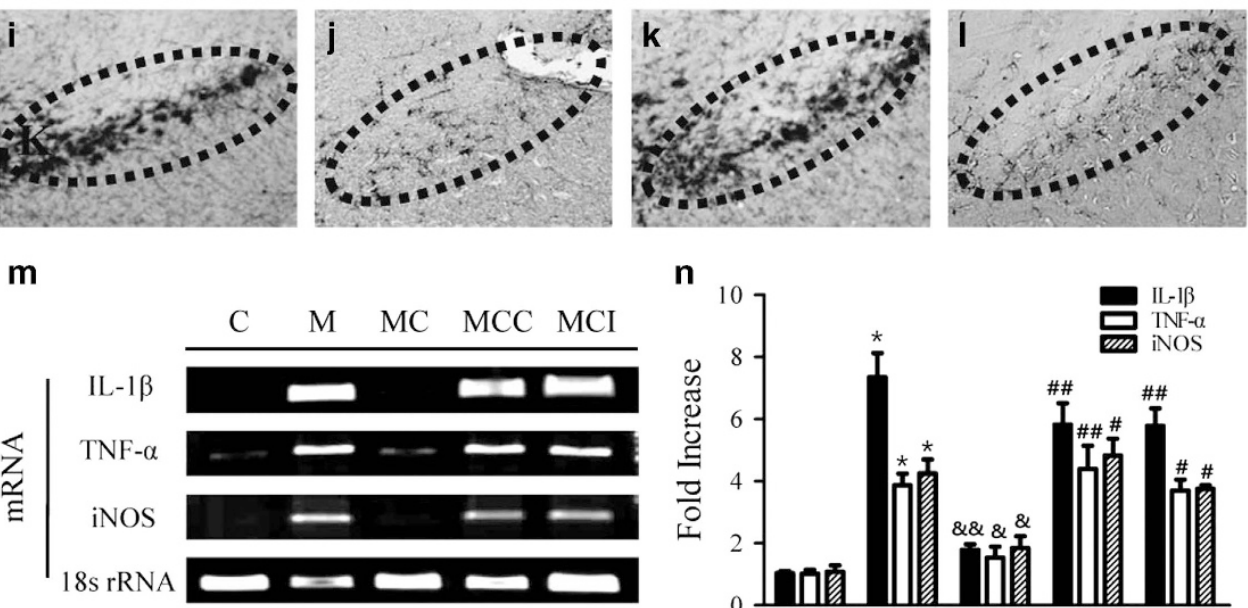

n

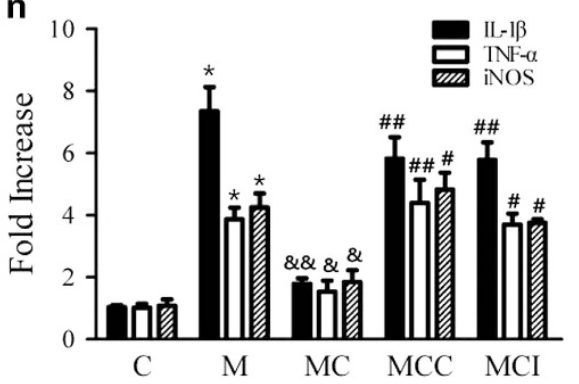

Figure 3 Capsaicin (CAP) blocks microglial activation and the expression of proinflammatory mediators in the substantia nigra (SN) in vivo. Animals receiving phosphate-buffered saline (PBS) as a control (a, b), CAP $\left(0.5 \mathrm{mg} \mathrm{kg}^{-1}\right)$ alone (c, d), 1-methyl-4-phenyl-1,2,3,6tetrahydropyridine (MPTP) (e, f), MPTP+CAP $(\mathbf{g}, \mathbf{h}), \operatorname{MPTP}+\mathrm{CAP}+\mathrm{CZP}(\mathbf{i}, \mathbf{j})$ and MPTP+CAP+I-RTX (k, I) were killed 3 days after the last MPTP injection. The brain tissues of the mice were removed and immunostained with antibodies for MAC-1 (a, $\mathbf{c}, \mathbf{e}, \mathbf{g}, \mathbf{i}, \mathbf{k})$ and ED-1 $(\mathbf{b}, \mathbf{d}, \mathbf{f}, \mathbf{h}, \mathbf{j}, \mathbf{l})$ to identify microglia. $n=5-7$. Dotted lines indicate SNpc. Scale bar, $100 \mu \mathrm{m}$. (m) Reverse transcription PCR showing the messenger RNA expression of proinflammatory cytokines in the SN. The total RNA was isolated from the SN at 1 day after the last injection of MPTP or vehicle in the absence or presence of CAP and/or CZP and I-RTX, TRPV1 antagonists. (n) Graphic representation of the means \pm s.e.m. of three to four samples. C, Control; M, MPTP; MC, MPTP and CAP; MCC, MPTP and CAP and CZP; MCI, MPTP and CAP and I-RTX. ${ }^{*} P<0.01$, significantly different from $C$. ${ }^{* *} P<0.01, \& P<0.05$, significantly different from $\mathrm{M}$. ${ }^{\#} P<0.05,{ }^{\#} P<0.01$, significantly different from MC (analysis of variance and Student-Newman-Keuls analysis). 
nigrostriatal DA neurons in the MPTP model of PD. ${ }^{8,21}$ Thus, we determined whether CAP might regulate the expression of IL-1 $\beta$, TNF- $\alpha$ and/or iNOS in the MPTP-lesioned SN, resulting in neuroprotection. The results of reverse transcription PCR showed that CAP attenuated MPTP-induced messenger RNA expression of IL- $1 \beta$ by $68 \% \quad(P<0.05)$, TNF- $\alpha$ by $52 \%$ $(P<0.01)$ and iNOS by $45 \%(P<0.01)$ in the SN (Figure $3 \mathrm{~m}$ and $n)$. The effects of CAP were reversed by treatment with CZP and I-RTX in the MPTP-lesioned SN (Figure $3 \mathrm{~m}$ and $\mathrm{n}$ ), suggesting TRPV1 involvement.
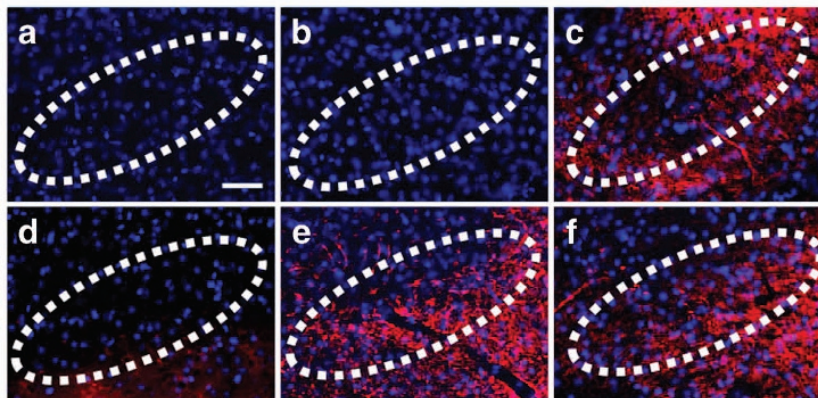

g
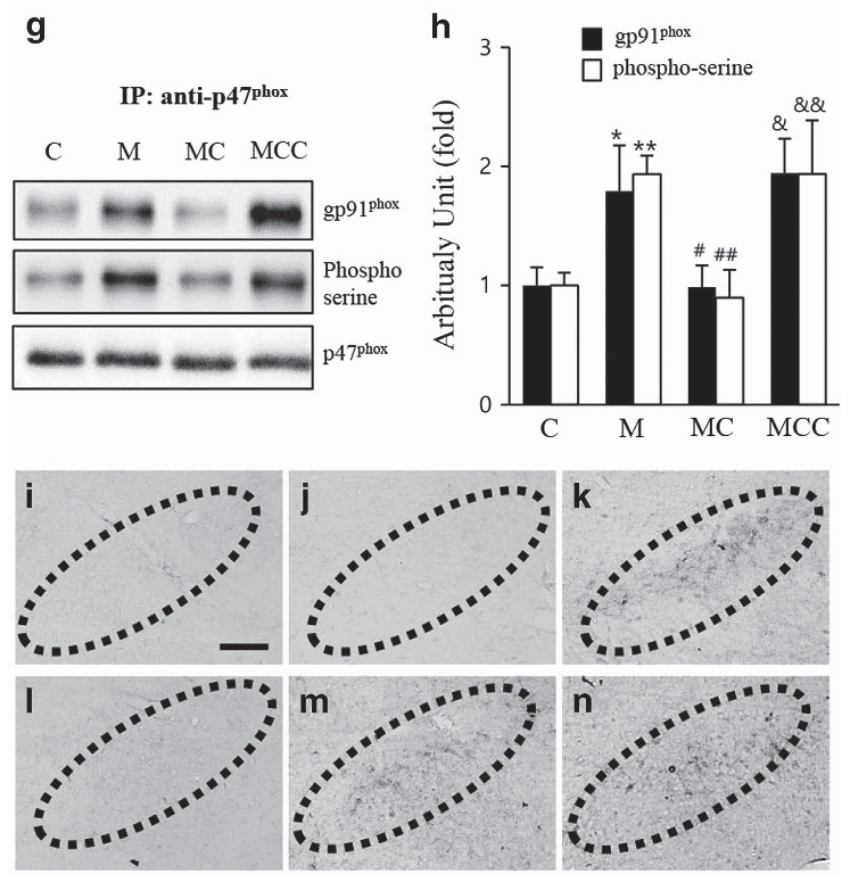

o

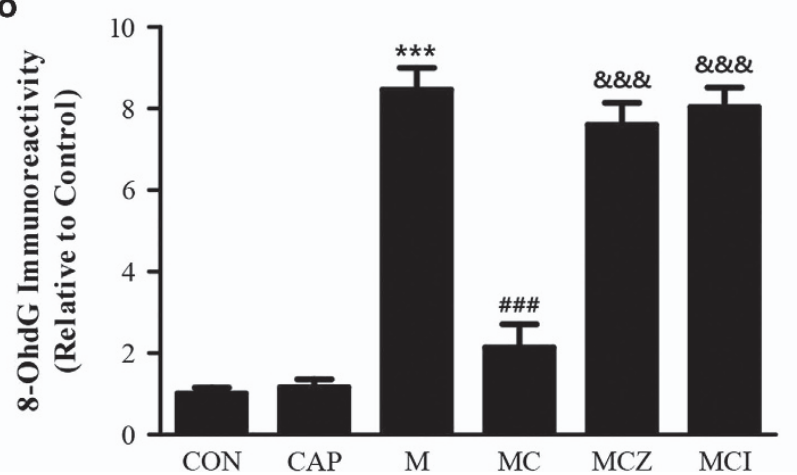

CAP reduces microglial NADPH oxidase-derived oxidative stress in the MPTP-lesioned $\mathrm{SN}$ in vivo

We recently demonstrated that MPTP induced microgliaderived production of ROS, such as hydrogen peroxide and superoxide, through NADPH oxidase activation, resulting in degeneration of DA neurons in the $\mathrm{SN} .{ }^{20,21,28}$ Accordingly, we examined the effect of CAP on MPTP-induced oxidant production in the SN. Hydroethidine histochemical staining revealed significant increases in ethidium (that is, fluorescent product of oxidized hydroethidine) in the MPTP-lesioned SN (Figure 4c) compared with PBS-treated control mice (Figure 4a). In the CAP-treated MPTP-lesioned SN, the in vivo production of oxidants was significantly reduced (Figure 4d), which was reversed by the TRPV1 antagonists CZP (Figure 4e) and I-RTX (Figure 4f), indicating TRPV1 involvement. As controls, CAP alone had no effect on the production of oxidants (Figure $4 \mathrm{~b}$ ).

Because microglial NADPH oxidase is a significant source of ROS in the MPTP-lesioned SN in vivo, ${ }^{6,7}$ we sought to determine whether CAP could exert neuroprotection by inhibiting NADPH oxidase-derived ROS production and the subsequent oxidative stress in the MPTP-lesioned $\mathrm{SN}^{22}$ Western blot analysis revealed that MPTP significantly upre-

Figure 4 Capsaicin (CAP) reduces 1-methyl-4-phenyl-1,2,3, 6-tetrahydropyridine (MPTP)-induced oxidant production and activation of NADPH oxidase in the substantia nigra (SN) in vivo. (a-f) Animals receiving phosphate-buffered saline (PBS) as a control (a), CAP (0.5 $\mathrm{mg} \mathrm{kg}^{-1}$ ) alone (b), MPTP (c), MPTP+CAP (d), MPTP $+C A P+C Z P$ (e) or MPTP+CAP+I-RTX (f) were killed 3 days after the last MPTP injection. SN tissues were prepared for hydroethidine histochemistry to detect oxidant production. Five to seven animals were used for each experimental group. Dotted lines indicate the SNpc. (g) Western blot analyses showed the phosphorylation of cytosolic subunits $\mathrm{p} 47^{\text {phox }}$ and $\mathrm{p} 47^{\text {phox }}$-gp $9^{\text {phox }}$ binding 3 days after MPTP injection, indicating activation of NADPH oxidase in the SN. The animals were killed 3 days after the last MPTP administration, and the SN tissues were isolated. Tissue lysates were immunoprecipitated with an anti-p47phox antibody and analyzed by immunoblotting with antibodies against $\mathrm{p} 47^{\text {phox }}$, gp91 ${ }^{\text {phox }}$ and phospho-serine. The total p47phox level indicates protein-loading control. (h) The graph represents phospho-serine and gp91phox levels expressed relative to the total p47phox. Means \pm s.e.m. of four samples. ${ }^{*} P<0.01,{ }^{* *} P<0.001$, compared with PBS; $\# P<0.01$, \#\# $P<0.001$, compared with MPTP only; $\& P<0.01, \& \& P<0.001$, compared with MPTP and CAP. (i-n) The $\mathrm{SN}$ tissues obtained from the same animals used in a-f were prepared for 8-OHdG immunostaining to detect oxidative damage in the SN. Control (i), CAP $\left(0.5 \mathrm{mg} \mathrm{kg}^{-1}\right)$ alone (j), MPTP (k), MPTP+CAP (I), MPTP+CAP+CZP (m) or MPTP+CAP+I-RTX (n). (o) Immunoreactivity of 8-OhdG in the $\mathrm{SN}$ was measured using Image J. $n=5-7$. Note that CAP prevented MPTP-induced DNA damage in the SN, which was reversed by the TRPV1 antagonists (CZP or I-RTX). C, Control; CAP, capsaicin; M, MPTP; MC, MPTP $+\mathrm{CAP}$; MCC, MPTP+CAP+CZP. ${ }^{* * *} P<0.001$, significantly different from controls; \#\#\# $P<0.001$, significantly different from MPTP only, $\& \& \& P<0.001$, significantly different from MC (analysis of variance and Student-Newman-Keuls analysis). 
gulated the levels of both $\mathrm{p} 47^{\text {phox }}$ phosphorylation and

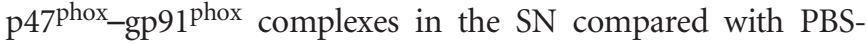
treated controls (Figure $4 \mathrm{~g}$ and $\mathrm{h}$ ), indicating the activation of $\mathrm{NADPH}$ oxidase. In the CAP-treated MPTP-lesioned SN, the levels of both $\mathrm{p} 47^{\text {phox }}$ phosphorylation and the $\mathrm{p} 47^{\text {phox }}$ gp91 $1^{\text {phox }}$ complex were significantly decreased compared with the levels in the SN of mice treated with MPTP alone (Figure $4 \mathrm{~g}$ and $\mathrm{h}$ ). These effects of CAP were reversed by CZP (Figure $4 \mathrm{~g}$ and $\mathrm{h}$ ). CAP alone did not affect the levels of phospho-

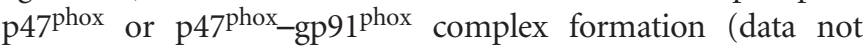
shown).

To explore CAP effects on oxidative stress to DNA in the MPTP-lesioned SN in vivo, we measured the levels of 8-OHdG, a marker of oxidative nucleic acid damage ${ }^{21,22}$ using immunostaining (Figure 4a-O). 8-OHdG-immunopositive cells were significantly increased in the $\mathrm{SN}$ at 3 days post MPTP $(P<0.001$; Figure $4 \mathrm{k})$ compared with the PBS-treated SN (Figure 4i). In the CAP-treated MPTP-lesioned SN, the oxidative DNA damage was markedly attenuated $(P<0.001$; Figure 41), which was reversed by the TRPV1 antagonists, CZP $(P<0.001$; Figure $4 \mathrm{~m})$ and I-RTX $(P<0.001$; Figure $4 \mathrm{n})$, indicating TRPV1 involvement. As controls, CAP alone did not affect DNA damage (Figure 4j).

\section{CAP inhibits astroglial MPO expression in the MPTP-}

\section{lesioned $\mathrm{SN}$ in vivo}

We recently reported that MPTP induced MPO expression in astrocytes in the $\mathrm{SN}$ in vivo. ${ }^{21,22}$ Given the reduced MPTP neurotoxicity in MPO-deficient mice, ${ }^{4}$ we wondered whether CAP could exert neuroprotection by modulating astroglial activation and MPO expression in the MPTP-lesioned SN in vivo. The immunohistochemical analysis revealed a significant increase in the expression of GFAP-labeled reactive astrocytes in the MPTP-lesioned SN (Figure 5b) compared with the PBS-treated SN (Figure 5a). In the CAP-treated MPTP-lesioned SN, few GFAP-labeled reactive astrocytes were expressed (Figure $5 \mathrm{c}$ ). These effects of CAP were reversed by CZP (Figure 5d) and I-RTX (Figure 5e). In line with our recent findings, ${ }^{21,22}$ immunohistochemical staining showed a significant increase in the expression of MPO-positive cells in the MPTP-lesioned SN (Figure 5g) compared with the PBS-treated control SN (Figure 5f). In the CAP-treated MPTP-lesioned SN, few MPO-positive cells were expressed (Figure 5h). Stereological counting of MPO-positive cells in the SN supported these results, revealing 279 -fold increases in the number of MPO-positive cells in the MPTP-lesioned SN compared with the PBS-treated control SN (Figure 5k). CAP significantly decreased the number of MPO-positive cells by $65 \%$ $(P<0.01$; Figure $5 \mathrm{k})$, which was reversed by CZP and I-RTX (Figure 5i-k).

\section{DISCUSSION}

In the present study, we demonstrated the in vivo effects of CAP in the MPTP mouse model of PD. TRPV1 activation by CAP inhibits brain inflammation and oxidative stress, which rescues DA neurons and improves motor deficits in the MPTP
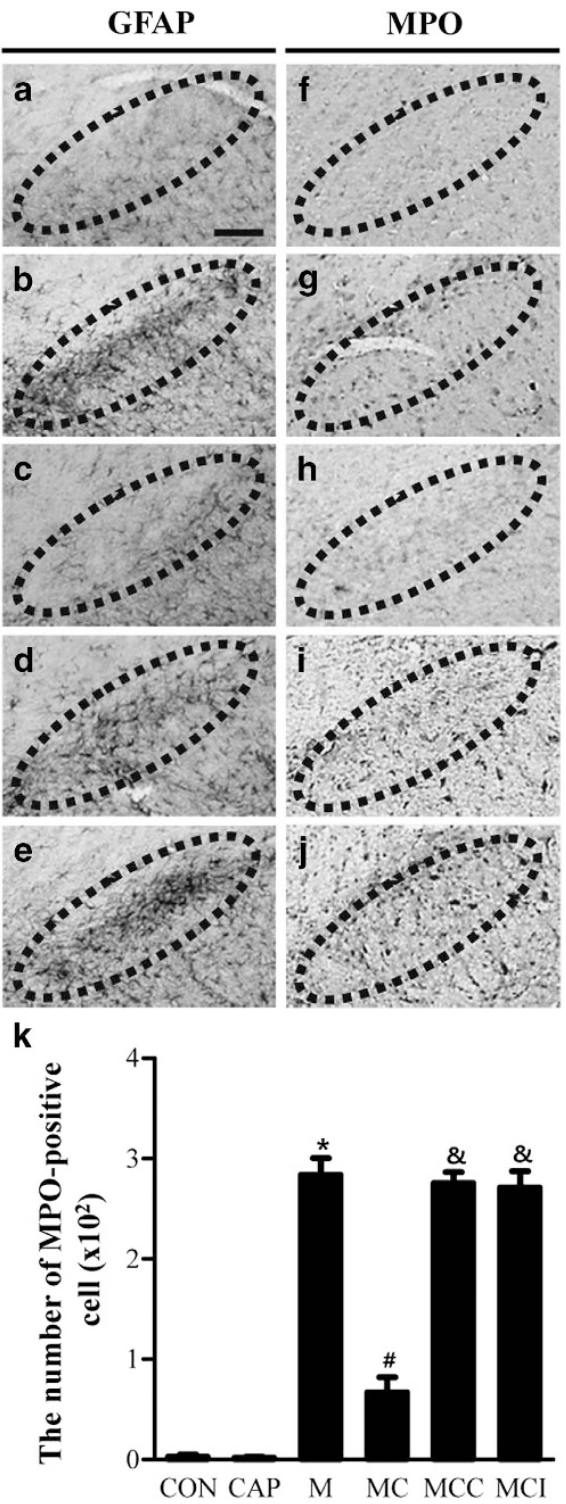

Figure 5 Capsaicin (CAP) inhibits 1-methyl-4-phenyl-1,2,3,6tetrahydropyridine (MPTP)-induced astroglial activation and the expression of MPO in the substantia nigra (SN) in vivo. The SN tissues obtained from the same animals as used in Figure 3 were immunostained with a GFAP antibody for astrocytes (a-e) and an MPO antibody for myeloperoxidase immunoreactivity $(\mathbf{f}-\mathbf{j})$. Animals that received PBS as a control (a, f), MPTP (b, g), MPTP+CAP $(\mathbf{c}, \mathbf{h}), \mathrm{MPTP}+\mathrm{CAP}+\mathrm{CZP}(\mathbf{d}, \mathbf{i})$ or MPTP+CAP+I-RTX $(\mathbf{e}, \mathbf{j})$ were killed 3 days after the last MPTP injection. Dotted lines indicate the SNpc. (k) The number of MPO-positive cells in the SN was counted. $n=5-7$. C, control; CAP, capsaicin; M, MPTP; MC, MPTP and CAP; MCC, MPTP and CAP and CZP; MCI, MPTP and CAP and I-RTX. ${ }^{*} P<0.01$, significantly different from controls; ${ }^{*} P<0.01$, significantly different from MPTP only, $\& P<0.01$, significantly different from MC (analysis of variance and Student-Newman-Keuls analysis).

mouse model of PD. Accompanying neuroprotection and behavioral recovery, CAP not only inhibited NADPH oxidase and astroglial MPO but it also decreased ROS/RNS production and oxidative damage to DNA. The beneficial effects of CAP 
were reversed by the TRPV1 antagonists, CZP and/or I-RTX, providing evidence for the involvement of TRPV1 in CAP neuroprotective events.

Glial cells comprising microglia and astrocytes are generally beneficial to neuronal functions in the brain. However, under neuropathological conditions, reactive glial cells are the primary producers of several potentially neurotoxic factors, including ROS/RNS and/or proinflammatory cytokines, ${ }^{4,7,22,29,30}$ ultimately causing neurodegeneration.

Reactive microglia can cause degeneration of DA neurons in the SN during inflammation by producing NADPH oxidasederived ROS. ${ }^{31,32}$ Many clinical and experimental data have demonstrated the existence of reactive microglia and increased ROS generation by activated NADPH oxidase originating from reactive microglia in the $\mathrm{SN}$ of $\mathrm{PD}$ patients ${ }^{33,34}$ and in the $\mathrm{SN}$ of the MPTP mouse PD model. ${ }^{21,22,35}$ NADPH oxidase is an enzyme that catalyzes ROS production and consists of the cytosolic component p47phox and the membrane component gp91phox. ${ }^{36,37}$ NADPH oxidase activation can be assessed by the increased levels of both $\mathrm{p} 47$ phox phosphorylation and p47phox-gp91phox complexes, ${ }^{38}$ which are the consequences of upregulated ROS. ${ }^{6,7,21}$ In the SN of both PD patients and MPTP-treated PD model mice, the levels of oxidative damage to DNA are significantly increased. ${ }^{21,39}$ Similar to our recent data, ${ }^{22}$ the activation of NADPH oxidase was evident in the MPTP-treated SN, which leads to increased ROS production and results in DNA damage, as visualized by hydroethidine staining and $8-\mathrm{OHdG}$ immunostaining in the $\mathrm{SN}$, respectively. ${ }^{20-22}$ In CAP-treated MPTP-lesioned SN, there was a decrease in NADPH oxidase activation, ROS production and oxidative damage on nucleic acid, which could be reversed by TRPV1 receptor antagonists. Our results collectively suggest that CAP contributes to neuroprotection in the MPTP-treated $\mathrm{SN}$ by blocking activation of NADPH oxidase and oxidative damage via TRPV1.

Microglia-derived inflammatory factors may trigger death of nigrostriatal DA neurons. Among such inflammatory factors capable of inducing neurodegeneration are NOS $^{8}$ and proinflammatory cytokines, including TNF- $\alpha$ and IL- $1 \beta .{ }^{40}$ These inflammatory mediators are increased in the brains of PD patients and are responsible for the degeneration of DA neurons in the MPTP-treated SN. ${ }^{21,22}$ The present results demonstrate that in the MPTP-treated $\mathrm{SN}$, the expression levels of iNOS, TNF- $\alpha$ and IL- $1 \beta$ messenger RNA are increased. In the CAP-treated MPTP-lesioned SN, however, the expression of these three molecules was attenuated, which could be reversed by TRPV1 antagonists, indicative of TRPV1-induced neuroprotection. A recent report demonstrated that SA13353, a TRPV1 agonist, inhibited LPS-induced TNF- $\alpha$ and IL- $1 \beta$ production in vivo and reduced the production of inflammatory cytokines in an EAE mouse model. ${ }^{41}$ Moreover, SA13353 prevented ischemic injury through suppression of TNF- $\alpha$ messenger RNA expression. ${ }^{42}$ Collectively, these results suggest that TRPV1 activation by CAP rescues nigrostriatal DA neurons in the MPTP-lesioned SN by reducing MPTPinduced expression of iNOS and proinflammatory cytokines.
Reactive microglia are not the only harmful factor to DA neurons in the MPTP mouse model of PD. Astrocytes, when activated, produce neurotoxic factors and are detrimental to the survival of DA neurons. ${ }^{4,22,29,30}$ Among them, reactive astrocyte-derived MPO is a significant source of cytotoxic ROS/RNS, which cause death of DA neurons in the MPTPlesioned SN. ${ }^{21,22}$ This can be observed in the SN of PD patients and of the MPTP mouse model of PD. ${ }^{4}$ Alternatively, MPO secretion can cause damage to nigral DA neurons via microglial activation. ${ }^{43}$ There was a significant increase in the expression of both MPO+ and GFAP+ cells in the MPTP-treated as analyzed by immunostaining. In the CAP-treated MPTPlesioned $\mathrm{SN}$, the number of $\mathrm{MPO}+$ cells was significantly decreased, which could be reversed by TRPV1 antagonists. The current findings suggest that TRPV1 activation by CAP rescues DA neurons by inhibiting astroglial MPO expression and the resultant oxidative damage in the MPTP-lesioned SN.

In summary, the present study shows that TRPV1 activation by CAP suppresses glial-derived oxidative stress and rescues DA neurons in the MPTP-lesioned SN. Taken together with our recent data, we tentatively propose that TRPV1 may be a possible target for the treatment of PD associated with neuroinflammation and oxidative stress.

\section{CONFLICT OF INTEREST}

The authors declare no conflict of interest.

\section{ACKNOWLEDGEMENTS}

This work was supported by a National Research Foundation of Korea (NRF) grant funded by the Korean government (MSIP; nos 2008-0061888, and 2014R1A1A2056508 and 2014R1A1A3051276).

1 Savitt JM, Dawson VL, Dawson TM. Diagnosis and treatment of Parkinson disease: molecules to medicine. J Clin Invest 2006; 116: 1744-1754.

2 Dauer W, Przedborski S. Parkinson's disease: mechanisms and models. Neuron 2003; 39: 889-909.

3 Seol W. Biochemical and molecular features of LRRK2 and its pathophysiological roles in Parkinson's disease. BMB Rep 2010; 43: 233-244.

4 Choi DK, Pennathur S, Perier C, Tieu K, Teismann P, Wu DC et al. Ablation of the inflammatory enzyme myeloperoxidase mitigates features of Parkinson's disease in mice. J Neurosci 2005; 25: 6594-6600.

5 Gao HM, Liu B, Zhang W, Hong JS. Critical role of microglial NADPH oxidase-derived free radicals in the in vitro MPTP model of Parkinson's disease. FASEB J 2003; 17: 1954-1956.

6 Wu DC, Teismann P, Tieu K, Vila M, Jackson-Lewis V, Ischiropoulos H et al. NADPH oxidase mediates oxidative stress in the 1-methyl-4-phenyl1,2,3,6-tetrahydropyridine model of Parkinson's disease. Proc Natl Acad Sci USA 2003; 100: 6145-6150.

7 Chung YC, Ko HW, Bok E, Park ES, Huh SH, Nam JH et al. The role of neuroinflammation on the pathogenesis of Parkinson's disease. BMB Rep 2010; 43: 225-232.

8 Liberatore GT, Jackson-Lewis V, Vukosavic S, Mandir AS, Vila M, McAuliffe WG et al. Inducible nitric oxide synthase stimulates dopaminergic neurodegeneration in the MPTP model of Parkinson disease. Nat Med 1999; 5: 1403-1409.

9 Wu DC, Jackson-Lewis V, Vila M, Tieu K, Teismann P, Vadseth C et al. Blockade of microglial activation is neuroprotective in the 1-methyl-4phenyl-1,2,3,6-tetrahydropyridine mouse model of Parkinson disease. J Neurosci 2002; 22: 1763-1771. 
10 Gunthorpe MJ, Szallasi A. Peripheral TRPV1 receptors as targets for drug development: new molecules and mechanisms. Curr Pharm/ Des 2008; 14 32-41.

11 Starowicz K, Cristino L, Di Marzo V. TRPV1 receptors in the central nervous system: potential for previously unforeseen therapeutic applications. Curr Pharm Des 2008; 14: 42-54.

12 Kauer JA, Gibson HE. Hot flash: TRPV channels in the brain. Trends Neurosci 2009; 32: 215-224.

13 Morgese MG, Cassano T, Cuomo V, Giuffrida A. Anti-dyskinetic effects of cannabinoids in a rat model of Parkinson's disease: role of $\mathrm{CB}(1)$ and TRPV1 receptors. Exp Neurol 2007; 208: 110-119.

14 Gonzalez-Aparicio R, Moratalla R. Oleoylethanolamide reduces L-DOPAinduced dyskinesia via TRPV1 receptor in a mouse model of Parkinson s disease. Neurobiol Dis 2014; 62: 416-425.

15 Park ES, Kim SR, Jin BK. Transient receptor potential vanilloid subtype 1 contributes to mesencephalic dopaminergic neuronal survival by inhibiting microglia-originated oxidative stress. Brain Res Bull 2012; 89: 92-96.

16 Marinelli S, Di Marzo V, Berretta N, Matias I, Maccarrone M, Bernardi G et al. Presynaptic facilitation of glutamatergic synapses to dopaminergic neurons of the rat substantia nigra by endogenous stimulation of vanilloid receptors. J Neurosci 2003; 23: 3136-3144.

17 de Lago E, de Miguel R, Lastres-Becker I, Ramos JA, Fernandez-Ruiz J. Involvement of vanilloid-like receptors in the effects of anandamide on motor behavior and nigrostriatal dopaminergic activity: in vivo and in vitro evidence. Brain Res 2004; 1007: 152-159.

18 Di Marzo V, Lastres-Becker I, Bisogno T, De Petrocellis L, Milone A Davis JB et al. Hypolocomotor effects in rats of capsaicin and two long chain capsaicin homologues. Eur J Pharmacol 2001; 420: 123-131.

19 Lee J, Di Marzo V, Brotchie JM. A role for vanilloid receptor 1 (TRPV1) and endocannabinnoid signalling in the regulation of spontaneous and L-DOPA induced locomotion in normal and reserpine-treated rats. Neuropharmacology 2006; 51: 557-565.

20 Chung YC, Bok E, Huh SH, Park JY, Yoon SH, Kim SR et al. Cannabinoid receptor type 1 protects nigrostriatal dopaminergic neurons against MPTP neurotoxicity by inhibiting microglial activation. J Immunol 2011; 187: 6508-6517.

21 Chung YC, Kim SR, Jin BK. Paroxetine prevents loss of nigrostriatal dopaminergic neurons by inhibiting brain inflammation and oxidative stress in an experimental model of Parkinson's disease. J Immunol 2010; 185: 1230-1237.

22 Huh SH, Chung YC, Piao Y, Jin MY, Son HJ, Yoon NS et al. Ethyl pyruvate rescues nigrostriatal dopaminergic neurons by regulating glial activation in a mouse model of Parkinson's disease. J Immunol 2011; 187: 960-969.

23 Giovanni A, Sieber BA, Heikkila RE, Sonsalla PK. Correlation between the neostriatal content of the 1-methyl-4-phenylpyridinium species and dopaminergic neurotoxicity following 1-methyl-4-phenyl-1,2,3,6-tetrahydropyridine administration to several strains of mice. J Pharmacol Exp Ther 1991; 257: 691-697.

24 Przedborski S, Jackson-Lewis V, Djaldetti R, Liberatore G, Vila M, Vukosavic $S$ et al. The parkinsonian toxin MPTP: action and mechanism. Restor Neurol Neurosci 2000; 16: 135-142.

25 Block ML, Zecca L, Hong JS. Microglia-mediated neurotoxicity: uncovering the molecular mechanisms. Nat Rev Neurosci 2007; 8: 57-69.

26 Henkel JS, Beers DR, Siklos L, Appel SH. The chemokine MCP-1 and the dendritic and myeloid cells it attracts are increased in the MSOD1 mouse model of ALS. Mol Cell Neurosci 2006; 31: 427-437.

27 Travaglione S, Falzano L, Fabbri A, Stringaro A, Fais S, Fiorentini C. Epithelial cells and expression of the phagocytic marker CD68: scavenging of apoptotic bodies following Rho activation. Toxicol In Vitro 2002; 16: 405-411.
28 Chung YC, Kim SR, Park JY, Chung ES, Park KW, Won SY et al. Fluoxetine prevents MPTP-induced loss of dopaminergic neurons by inhibiting microglial activation. Neuropharmacology 2011; 60: 963-974.

29 Bolin LM, Strycharska-Orczyk I, Murray R, Langston JW, Di Monte D. Increased vulnerability of dopaminergic neurons in MPTP-lesioned interleukin-6 deficient mice. J Neurochem 2002; 83: 167-175.

30 Oki C, Watanabe Y, Yokoyama H, Shimoda T, Kato H, Araki T. Delayed treatment with arundic acid reduces the MPTP-induced neurotoxicity in mice. Cell Mol Neurobiol 2008; 28: 417-430.

31 Choi SH, Lee DY, Chung ES, Hong YB, Kim SU, Jin BK. Inhibition of thrombin-induced microglial activation and NADPH oxidase by minocycline protects dopaminergic neurons in the substantia nigra in vivo. $J$ Neurochem 2005; 95: 1755-1765.

32 Choi SH, Lee DY, Kim SU, Jin BK. Thrombin-induced oxidative stress contributes to the death of hippocampal neurons in vivo: role of microglial NADPH oxidase. J Neurosci 2005; 25: 4082-4090.

33 McGeer PL, McGeer EG. Glial reactions in Parkinson's disease. Mov Disord 2008; 23: 474-483.

34 Mirza B, Hadberg $H$, Thomsen $P$, Moos $T$. The absence of reactive astrocytosis is indicative of a unique inflammatory process in Parkinson's disease. Neuroscience 2000; 95: 425-432.

35 Vroon A, Drukarch B, Bol JG, Cras P, Breve JJ, Allan SM et al. Neuroinflammation in Parkinson's patients and MPTP-treated mice is not restricted to the nigrostriatal system: microgliosis and differential expression of interleukin-1 receptors in the olfactory bulb. Exp Gerontol 2007; 42: 762-771.

36 Babior BM. NADPH oxidase: an update. Blood 1999; 93: 1464-1476.

37 Cross AR, Segal AW. The NADPH oxidase of professional phagocytesprototype of the NOX electron transport chain systems. Biochim Biophys Acta 2004; 1657: 1-22.

38 Fan J, Li Y, Levy RM, Fan JJ, Hackam DJ, Vodovotz Y et al. Hemorrhagic shock induces NAD(P)H oxidase activation in neutrophils: role of HMGB1TLR4 signaling. J Immunol 2007; 178: 6573-6580.

39 Nakabeppu Y, Tsuchimoto D, Yamaguchi H, Sakumi K. Oxidative damage in nucleic acids and Parkinson's disease. J Neurosci Res 2007; 85: 919-934.

40 Nagatsu T, Mogi M, Ichinose H, Togari A. Cytokines in Parkinson's disease. J Neural Transm Suppl 2000; 58: 143-151.

41 Tsuji F, Murai M, Oki K, Seki I, Ueda K, Inoue H et al. Transient receptor potential vanilloid 1 agonists as candidates for anti-inflammatory and immunomodulatory agents. Eur J Pharmacol 2010; 627: 332-339.

42 Ueda K, Tsuji F, Hirata T, Murai M, Aono H, Takaoka M et al. Preventive effect of SA13353 [1-[2-(1-adamantyl)ethyl]-1-pentyl-3-[3-(4-pyridyl) propyl]urea], a novel transient receptor potential vanilloid 1 agonist, on ischemia/reperfusion-induced renal injury in rats. J Pharmacol Exp Ther 2009; 329: 202-209.

43 Lau D, Mollnau H, Eiserich JP, Freeman BA, Daiber A, Gehling UM et al. Myeloperoxidase mediates neutrophil activation by association with $\mathrm{CD} 1 \mathrm{lb} /$ CD18 integrins. Proc Natl Acad Sci USA 2005; 102: 431-436.

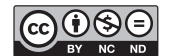

This work is licensed under a Creative Commons Attribution-NonCommercial-NoDerivs 4.0 International License. The images or other third party material in this article are included in the article's Creative Commons license, unless indicated otherwise in the credit line; if the material is not included under the Creative Commons license, users will need to obtain permission from the license holder to reproduce the material. To view a copy of this license, visit http://creativecommons.org/licenses/by-nc-nd/4.0/

Supplementary Information accompanies the paper on Experimental \& Molecular Medicine website (http://www.nature.com/emm) 